\title{
Introduction to a special issue on the neuroscience of motivation and emotion
}

\author{
Eddie Harmon-Jones · Jack van Honk
}

Published online: 28 February 2012

(C) Springer Science+Business Media, LLC 2012

Neuroscience methods have gained widespread use in many areas of psychological and behavioral sciences. Their use has likewise increased in research and theory on motivation and emotion. As a consequence of this explosion of interest, we organized this special issue. In organizing this issue, we thought it important to have contributions from a range of neuroscience methods, because addressing issues concerning motivation and emotion requires multiple methods, each with their own benefits and limitations, particularly when used in studying emotive processes in humans. In addition, we invited scientists to contribute who are making programmatic neuroscientific contributions to the study of motivation and emotion, and were pleased that all invited agreed to contribute.

The study of physiological and biological processes in motivation and emotion dates back several decades in nonhuman animal research and human research. Indeed, scientists studying motivation and emotion were some of the first to employ physiological measurements in their work. Consider Walter Cannon's (1915) classic experiments on the fight or flight response, and Albert Ax's (1953) classic experiment elucidating physiological differences between

\footnotetext{
E. Harmon-Jones $(\bowtie)$

University of New South Wales, Sydney, NSW, Australia

e-mail: eddiehj@gmail.com

J. van Honk

Utrecht University, Utrecht, The Netherlands

J. van Honk

Cape Town University, Cape Town, South Africa
}

anger and fear. However, different names of the field(s) employing these methods and models have been used over time. The field has been referred to as biological psychology, psychobiology, physiological psychology, neuropsychology, psychophysiology, as well as other names. Although some of these terms are still used to describe specific types of conceptual and/or methodological approaches, in our view, these fields are all associated with neuroscience, the term most commonly used today in describing research into physiological and biological processes involving brain/mind.

The explosion of interest in neuroscience within the study of motivation, emotion, and other psychological processes is likely due to several factors. One prominent factor contributed to the explosion of interest is the advent of new and more readily available methods. For example, the signal of most interest in functional magnetic resonance imaging, the blood oxygenation level dependent (BOLD) signal, was first successfully measured in the human brain in the early 1990s (Kwong et al. 1992), and it has since gained widespread research usage. Also, within the last decade, several companies produced easy-to-use acquisition and signal processing systems for use with physiological responses such as electroencephalography (EEG), event-related brain potentials (ERPs), and electromyography. At the same time, the growing interest in the neuroscience of human motivation and emotion coincided with the advent of salivary measurement and methods for acute single administrations of the hormones oxytocin and testosterone, and in research these hormones subsequently proved to be critical motivators for numerous human social and affective behaviors (Bos et al. in press).

Emotive neuroscience integrates diverse literatures, theories, and methodologies to address questions about brain, mind, and behavior and thus creates a science 
presumably closer to yielding answers to important questions. This interdisciplinary research approach can potentially provide a bridge to other knowledge that may ultimately help to explain or better understand a condition or behavior. For example, activity in a brain region may be associated with other behaviors, neurotransmitters, or hormones that may in turn might shed light on the original behavior or condition of interest (Carver and HarmonJones 2009). Also, neuroscience methods can be used in tests of theoretical interpretations of behavioral effects, as several papers in this special issue illustrate.

In addition, neuroscience methods provide sources of information that subvert some of the problems with selfreport and other behavioral measures. For example, measures of brain activity, such as EEG, ERP, and fMRI, allow researchers to record rapid, online changes in motivational and affective responses that would otherwise be impossible to assess without interrupting a participant's engagement in an experimental manipulation or impossible to assess because these processes are not available in consciousness.

As noted above, the contributors, who are making important programmatic neuroscientific contributions to the study of motivation and emotion, employ a range of neuroscience methods and address a range of questions related to motivation and emotion. We briefly review their contributions below.

Jaak Panksepp, a pioneer in the field who coined the term affective neuroscience, leads off with an essay pointing to the necessity of studying primary emotional feelings in non-humans. He differentiates these processes from secondary-processes concerning learning and memory and tertiary processes concerning cognitive thinking and rumination. In the end, he posits that this conceptual view integrates basic and dimensional approaches to emotions.

Alexander Todorov reviews findings from primate and human neuroscience on face processing and the amygdala. He considers faces as imperative stimuli, and suggests that cognition, affect and motivation intersect in face perception. One of the key functions of the amygdala would be to direct attention to faces that are atypical or ambiguous. Todorov's in-depth framework is also consistent with amygdala findings that do not involve faces, and is therefore a universal account for the role of the amygdala in perception.

Tom Price and Eddie Harmon-Jones review research suggesting that manipulated facial expressions, hand contractions, and changes in physical posture influence approach motivation or the inclination to move toward a stimulus as assessed by physiological measures (i.e., asymmetric EEG alpha power over the frontal cortex, the late positive potential of the ERP, and the startle eyeblink response). They conclude that bi-directionality may exist between certain bodily movements and other components of approach- or avoidance-related emotions.

Jennifer Beer reviews recent research on motivated social cognition. Her review reveals what can be learned by examining motivational influences on the neural systems underlying social cognition. In particular, her review suggests that unrealistically positive evaluations of oneself and one's close other causes reduced orbitofrontal cortex activation compared to evaluations of others. Thus, these results contribute to the debate over whether unrealistic positivity reflects active distortion or cognitive conservation and they are more consistent with the cognitive miser perspective.

Dennis Schutter and Gennady Knyazev expertly review electrophysiological studies on the relations between motivation, emotion and cross-frequency coupling of brain oscillations. They suggest, on basis of this evidence, that the study of interdependencies of brain oscillations may be a valuable approach for studying processes associated with motivation and emotion. For instance, amplitude-amplitude coupling between delta-alpha and delta-beta is associated with state anxiety and approach-avoidance-related motivation. Also, the coupling of delta-beta oscillations changes following successful psychotherapy.

John Jost and David Amodio provide a timely review integrating previous behavioural research on motives underlying political orientation with emotive neuroscience research concerned with reactions to uncertainty, ambiguity, threat, and disgust. Their review suggests that right(vs. left-) wing political orientation is associated with greater neural sensitivity to threat and a larger amygdala region, as well as less sensitivity to response conflict and a smaller anterior cingulate region.

Estrella Montoya, David Terburg, Peter Bos, and Jack van Honk put forward a framework for the interactive role of steroid hormones cortisol and testosterone and the monoamine serotonin in impulsive aggression. First focusing on steroid hormones, they review evidence that suggests that high testosterone-to-cortisol ratio sets a predisposition for social aggressive behaviour in general. Next, they review evidence that suggests that serotonin may differentiate between impulsive and instrumental aggression, in that low prefrontal serotonin synthesis in combination with a high testosterone-low cortisol ratio produces a socially explosive mind.

Alicia Salvador in her insightful review discusses the interactive role of the hypothalamic-pituitary-gonadal (HPG) axis and the hypothalamic-pituitary-adrenal (HPA) axis in a broad spectrum of social behaviors. The interactions between these axes and their end products, the hormones testosterone and cortisol, are highly adaptive in social situations that involve competition and challenge and have components of social stress. 
Anna Weinberg, Anja Riesel, and Greg Hajcak review over two decades of research and theories about the errorrelated negativity (ERN), a negative-going wave in the event-related brain potential that occurs following the commission of an error. After presenting and critically evaluating cognitive theories of the ERN, they review research that suggests that the ERN is neural index of a neurobehavioral trait and variation in its amplitude is partially related to individual differences in defensive reactivity.

Collectively, these papers illustrate the multifarious ways in which the inclusion of neuroscience can benefit the study of motivation and emotion. They demonstrate how neuroscience approaches can lead to better understandings of phenomena; generate new predictions and new theories, even at the behavioural level; inform established psychological theories; and be used in tests of competing theories.

\section{References}

Ax, A. F. (1953). The physiological differentiation between fear and anger in humans. Psychosomatic Medicine, 15, 433-442.

Bos, P. A., Panksepp, J., Bluthé, R.-M., \& van Honk, J. (in press). Acute effects of steroid hormones and neuropeptides on human social-emotional behavior: A review of single administration studies. Frontiers in Neuroendocrinology.

Cannon, W. B. (1915). Bodily changes in pain, hunger, fear and rage: An account of recent researches into the function of emotional excitement. New York, NY: D. Appleton \& Company.

Carver, C. S., \& Harmon-Jones, E. (2009). Anger is an approachrelated affect: Evidence and implications. Psychological Bulletin, 135, 183-204.

Kwong, K. K., Belliveau, J. W., Chesler, D. A., Goldberg, I. E., Weisskoff, R. M., Poncelet, B. P., et al. (1992). Dynamic magnetic resonance imaging of human brain activity during primary sensory stimulation. Proceedings of National Academy of Science USA, 89, 5675-5679. 